\title{
TV/Series
}

\section{La religion et les grandes questions de société : l'exemple de la représentation de la peine de mort dans les séries américaines}

\section{Julie Richard et Georges Caron}

\section{(e) OpenEdition \\ Journals}

Édition électronique

URL : http://journals.openedition.org/tvseries/444

DOI : $10.4000 /$ tvseries.444

ISSN : 2266-0909

Éditeur

GRIC - Groupe de recherche Identités et Cultures

Référence électronique

Julie Richard et Georges Caron, «La religion et les grandes questions de société : l'exemple de la représentation de la peine de mort dans les séries américaines », TV/Series [En ligne], 5 | 2014, mis en ligne le 01 mai 2014, consulté le 19 avril 2019. URL : http://journals.openedition.org/tvseries/444 ; DOI : 10.4000/tvseries.444

\section{(c) (i) () $\Theta$}

TV/Series est mis à disposition selon les termes de la licence Creative Commons Attribution - Pas d'Utilisation Commerciale - Pas de Modification 4.0 International. 


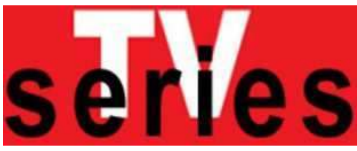

La religion et les grandes questions de société : l'exemple de la représentation de la peine de mort dans les séries américaines

Julie RICHARD et Georges CARON

La religion apparaît de manière courante dans les séries télévisées américaines, y compris dans des épisodes où la question religieuse n'est pas évoquée. Cependant, les épisodes qui traitent de la peine de mort permettent d'analyser le poids de la religion dans ce débat de société. L'étude de la représentation de la peine de mort dans plusieurs séries a permis d'identifier un discours dominant et ses exceptions. Les différents éléments religieux (ou « traces religieuses ») y occupent plusieurs fonctions : construction de « l'effet de réel », soutien moral et consolation, ritualisation voire sacralisation du protocole de l'exécution, arguments d'autorité et enfin, dispositif rhétorique et visuel. Les éléments religieux présents dans la représentation de la mise à mort et les arguments religieux échangés lors des débats sur la peine capitale servent à valider et/ou critiquer cette pratique. La religion apparaît également comme une instance de ritualisation et de légitimation, parmi d'autres. Ces différents indices pointent vers une sécularisation partielle et relative de cette question de société sur les écrans : les abolitionnistes utilisent peu les références religieuses et les valeurs religieuses sont davantage implicites qu'explicites.

$\mathrm{L}$ e premier épisode de la cinquième saison de la série The Good Wife (CBS, 2009-), dont la diffusion a débuté à la rentrée 2013, présente dans sa deuxième scène une exécution par injection létale, à laquelle assistent deux personnages principaux, les avocates chargées des derniers recours du condamné. Cette scène est d'autant plus frappante qu'elle commence sans explication, du point de vue des témoins, de l'autre côté de la vitre de la chambre d'exécution. De plus, le condamné, sanglé sur la table de mise à mort, est hissé dans une position verticale pour pouvoir délivrer ses derniers mots. Il apparaît ainsi, aux témoins et au spectateur, mis en croix. La scène tourne au cauchemar lorsque les derniers mots du crucifié sont interrompus : du sang gicle de son bras, la perfusion n'a pas tenu. Au cour d'une exécution, nous retrouvons donc une iconographie religieuse (figure 1). 


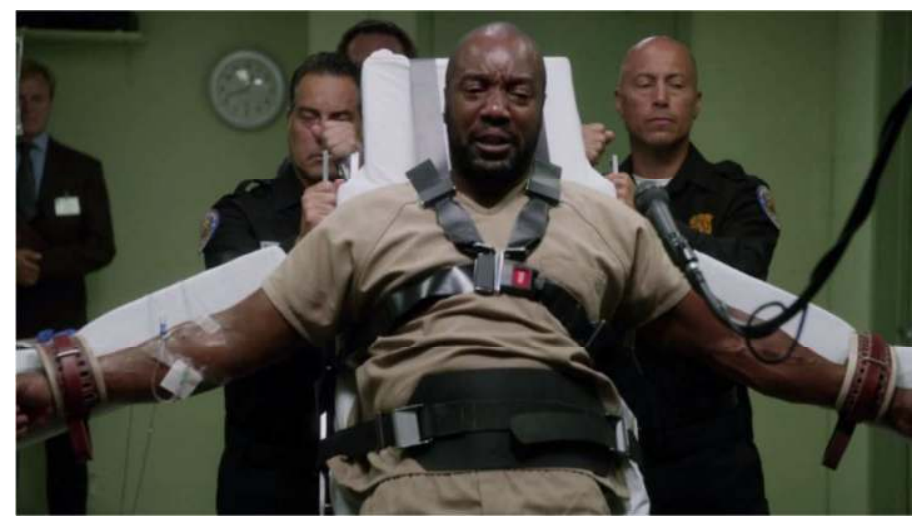

Fig. 1 : Une mise en croix à l'occasion d'une injection létale (The Good Wife, 5.1)

Cette image nous renvoie symboliquement à la place de la religion dans la société américaine, telle qu'elle est préjugée. En effet, cette société est souvent décrite comme particulièrement marquée par la religion. Dans ce pays, où les religions chrétiennes sont dominantes (les trois quarts de la population ${ }^{1}$ ), $65 \%$ des personnes interrogées déclarent que la religion tient une place importante dans leur vie quotidienne (contre $25 \%$ en France, par exemple ${ }^{2}$ ) et, en 2013, $87 \%$ des Américains déclarent croire en Dieu3. L'opinion publique américaine semble donc avoir échappé au mouvement de sécularisation ${ }^{4}$ connu par d'autres démocraties occidentales et libérales 5 .

Les grandes questions de société sont ainsi formulées en termes éthico-religieux (avortement, euthanasie, ou, plus récemment mariage gay $^{6}$ ), phénomène auquel le débat sur la peine de mort n'échappe pas. Ce débat se traduit notamment par un recul de cette pratique (handicapés mentaux et mineurs en sont aujourd'hui protégés), et une érosion de l'adhésion à la peine de mort dans les sondages, depuis la

\footnotetext{
Selon l'enquête de 2007 du Pew Research Center (http://religions.pewforum.org/affiliations consulté en février 2014)

2 Sondage Gallup, 2006-2008 (www.gallup.com/poll/114211/alabamians-iranianscommon.aspx consulté en septembre 2013)

3 Sondage Gallup, réalisé en mai 2013 (www.gallup.com/poll/169o/religion.aspx\#2, consulté en février 2014)

4 C'est-à-dire un processus d'autonomisation vis-à-vis de la religion et des valeurs traditionnellement défendues en son nom.

5 Camille Froidevaux-Metterie, Politique et religion aux États-Unis, Paris, La Découverte, 2009, p. 3 : « à rebours de la plupart des nations occidentales, les États-Unis se seraient soustraits au processus de sécularisation pour présenter le cas unique d'une démocratie ayant intégré la religion dans la sphère publique. »

6 Froidevaux-Metterie, p. 110: «Aux États-Unis, l'utilisation des symboles et du vocabulaire religieux fait partie intégrante de la vie politique. »
} 
deuxième moitié des années 1990 (en 2013, 60\% des sondés se sont déclarés favorables à la peine capitale7).

De plus, l'influence de la religion sur la société apparaît dans les productions culturelles: romans, films et séries télévisées. Ces dernières sont l'objet de cette étude, qui s'appuie sur un échantillon d'une vingtaine d'épisodes, diffusés pour la première fois entre 1990 et 2013. Nous avons extrait cet échantillon d'un corpus plus vaste, constitué dans le cadre d'une recherche sur la représentation de la peine de mort, dans toutes les dimensions évoquées à l'écran (judiciaires, raciales, sociales, symboliques, ...). Parmi les quelques 70 épisodes de ce corpus, nous avons sélectionné, dans la perspective de cet article, ceux où la religion tient une place suffisamment remarquable pour être repérée dans le formulaire de recueil de données mis au point pour cette première recherche. Un dernier critère de filtrage est le cadre contemporain : ont été conservés les épisodes dont les décors favorisent « l'effet de réel ${ }^{8}$ ». Par conséquent, les séries policières et carcérales forment l'essentiel des épisodes étudiés ${ }^{9}$.

La «mise en série » de ces épisodes nous a permis d'identifier un discours dominant et ses leitmotive. La religion y est présente à la fois comme élément de la vie sociale, du protocole de la mise à mort et du débat sur la peine de mort. Elle apparaît sous des formes diverses : citations bibliques, choix de la musique, présence de personnel religieux, ... ensemble d'éléments que nous appellerons «traces». Cependant, la religion a-t-elle une place prépondérante dans les représentations de cette grande question de société qu'est la peine de mort? C'est donc la question de la sécularisation que nous nous proposons d'étudier, grâce à une typologie des différentes fonctions occupées par les «traces religieuses" dans ces épisodes, en les remettant, en conclusion, dans le contexte du corpus général évoqué plus haut.

\section{Les « traces religieuses » et l'effet de réel}

Les « traces religieuses » entretiennent de multiples relations avec la réalité. Le fait religieux, dans sa diversité, contribue à construire l'effet de réel, et peut également être instrumentalisé à des fins narratives et/ou idéologiques.

\footnotetext{
${ }^{7}$ Sondage Gallup, réalisé en octobre 2013 (www.gallup.com/poll/1606/Death-Penalty.aspx) consulté en octobre 2013)

8 François Jost, De quoi les séries américaines sont-elles le symptôme?, Paris, CNRS Editions, 2012, p. 10 évoque la "familiarité de la fiction », qui « [comble] le fossé qui existe toujours entre notre monde et celui de la fiction. »

9 On y trouve également deux épisodes de séries fantastiques, où la présence du surnaturel coexiste avec un cadre de vie ordinaire.
} 
L'effet de réel est ainsi construit par l'utilisation de références connues. $O Z$ (HBO, 1997-2003) et Dernier recours $1.8^{10}$ (In Justice, $\mathrm{ABC}, 2006)$, par exemple, présentent des personnages de religieuses, ferventes abolitionnistes, agissant pour sauver la vie de condamnés, y compris coupables. Ces personnages sont des allusions à une personne réelle, Helen Prejean, nonne ayant accompagné cinq condamnés à mort, et connue pour son best-seller : Dead Man Walking, publié en $1993^{11}$. Le personnage non récurrent de la Sœur Quinn (Charlayne Woodard) dans Dernier recours peut ainsi être campé très rapidement par les scénaristes, grâce à cette allusion à une personne réelle célèbre.

L'effet de réel peut aussi s'appuyer sur une référence religieuse savante, ou apparaissant telle. C'est le cas pour le seul condamné à mort du corpus identifié comme musulman, que l'on trouve dans New York, unité spéciale 3.15 (Law and Order, Special Victims Unit, NBC, 1999-). La police a besoin de son aide, mais celui-ci n'a rien à y gagner. Comment justifier sa collaboration au spectateur ? La réponse apportée par les auteurs est religieuse : comme il est musulman, il va obéir au Coran, qui lui enjoint de payer sa «dette de sang 12 ». Répondre aux enquêteurs, à la demande du père de sa victime, fait partie de son chemin vers le rachat. Ce chemin n'aboutira cependant vraiment qu'avec son exécution, dont il souhaite avancer la date. Ce personnage est donc défini uniquement par sa religion, qui sert à faire avancer l'intrigue. Appuyés sur une référence au Coran, les scénaristes convoquent et confortent cependant un stéréotype : celui du musulman qui suit forcément à la lettre les prescriptions coraniques ${ }^{13}$.

Enfin, l'effet de réel peut découler d'une affirmation directe. Dans $O Z$ 6.8, le spectateur entend de la bouche du narrateur :

LE NARRATEUR. Laissez-moi vous raconter une petite histoire, ... une histoire vraie. L'année dernière, le juge de la Cour suprême des ÉtatsUnis, Anton Scalia, a critiqué la position de l'Église catholique sur la peine capitale. Il est convaincu que les juges catholiques qui sont opposés à la peine de mort devraient démissionner. Lui-même catholique et dévot, Scalia n'a pas exigé la démission des juges qui défendent la position de l'Église sur l'avortement ${ }^{14}$.

${ }^{10}$ Plusieurs épisodes d'une même série peuvent faire partie du corpus. Les épisodes, cités uniquement par le titre de leur série, font toujours référence au même épisode, sauf mention contraire.

${ }^{11}$ Le livre a été adapté au cinéma (Dead Man Walking, film américain, réalisé par Tim Robbins en 1995) et porté sur scène sous forme d'un opéra (Dead Man Walking, opéra de Jake Heggie, créé en 2000).

${ }^{12}$ Le Coran fait effectivement référence au « prix du sang », IV-92

${ }^{13}$ Une enquête réalisée en 2002 révèle que 40\% des personnes interrogées estiment que l'islam abrite des extrémistes plus violents que les autres religions, selon André Kaspi, François Durpaire, et alii, La civilisation américaine, Paris PUF, 2004, p. 254 ; l'épisode en question est diffusé pour la première fois en 2003.

${ }^{14}$ Les citations de dialogue sont des transcriptions et des traductions des auteurs. Dialogue d'origine : « Here's a story, and ... it's true. Last year, United States Supreme Court Justice 
Diffusé pour la première fois en février 2003, l'épisode fait allusion à des propos tenus par Justice Scalia lors d'une conférence organisée par le Pew Forum en janvier 2002 sur la peine de mort et la religion ${ }^{15}$, preuve s'il en faut, que le débat réel n'est pas complètement sécularisé. Malgré son apparente authenticité, cette anecdote simplifie les déclarations du juge de la Cour suprême. Celui-ci commente la nouvelle position de l'Église catholique sur la peine de mort. Au nom du respect de la vie, sont refusés la contraception, l'avortement, l'euthanasie, et l'usage de la peine de mort est déclaré inutile ${ }^{16}$. Selon le juriste, cette nouvelle position n'a pas la force d'un article de la foi, et n'oblige donc pas le fidèle. Scalia précise alors que si c'était le cas, les catholiques seraient exclus de la vie politique et judiciaire américaine :

[...] Je ne pense pas que cela serait une bonne chose si les catholiques américains se présentant à une fonction politique devaient s'opposer à la peine de mort. La plupart d'entre eux ne seraient pas élus. [...] Estil prudent de pousser, de fait, les catholiques à se retirer de la vie publique dans un pays où le gouvernement fédéral et 38 États fédérés, abritant environ $85 \%$ de la population, croient que la peine de mort est parfois juste et appropriée ${ }^{17}$ ?

La réalité est donc bien une source d'inspiration pour l'anecdote $\mathrm{du}$ narrateur. Cependant, celle-ci est le résultat de modifications effectuées dans un but abolitionniste. Elles présentent Justice Scalia comme un conservateur illogique, qui choisit de suivre les positions de son Église si elles correspondent à sa vision de la société.

\section{Les « traces religieuses » comme soutien moral et moyen de consolation}

Faire face à une exécution, situation par essence difficile, bouleverse car il s'agit d'une mort préméditée, organisée par la justice.

Anton Scalia criticized the Catholic Church's opposition to the death penalty. He believes Catholic judges who don't support capital punishment should resign. A devote Catholic himself, Scalia has not demanded judges who support the Church's stand on abortion should resign. "

${ }^{15} \mathrm{La}$ conférence, intitulée "Call for Reckoning: Religion and the Death Penalty ", s'est déroulée à Chicago (University of Chicago Divinity School) le 25 janvier 2002, voir www.features.pewforum.org/death-penalty/index.html

${ }^{16}$ Scalia commente l'encyclique Evangelium Vitae de Jean-Paul II, publiée le 25 mars 1995, et le catéchisme révisé par la suite.

17 « [...] I do not think it would be a good thing if American Catholics running for legislative office had to oppose the death penalty. Most of them would not be elected. [...] Is it prudent to effectively urge the retirement of Catholics from public life in a country where the federal government and 38 of the states, comprising about 85 percent of the population, believe the death penalty is sometimes just and appropriate? " (www.features.pewforum.org/deathpenalty/resources/transcript3.html consulté en janvier 2014) 
Devant cette épreuve, les personnages font appel à la religion, qui joue alors un de ses rôles traditionnels de soutien et de consolation.

Tout d'abord, dans de nombreux épisodes étudiés, le personnel religieux accompagne héros et condamnés. Ici, la figure récurrente est celle de l'aumônier de prison. Par exemple dans l'épisode 1.13 de la série Prison Break (FOX, 2005-2009), un soutien moral est apporté au frère du condamné à mort, Michael (Wentworth Miller), lui-même emprisonné, lors de l'épisode précédent l'exécution. L'aumônier (Tom McElroy) se rend dans sa cellule, discute, l'assure de sa présence le lendemain, jusqu'au dernier moment s'il le souhaite (figure 2).

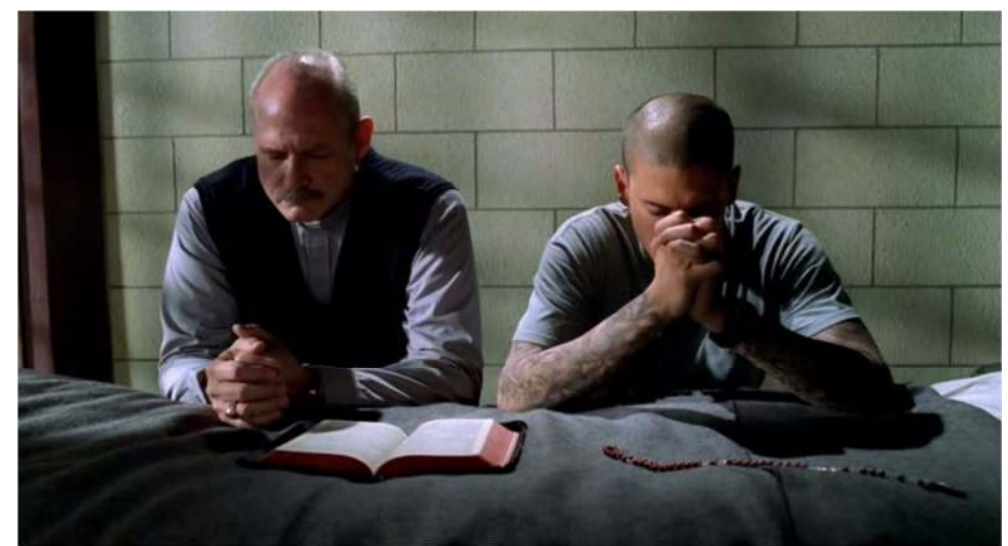

Fig. 2 : L’aumônier de prison et Michael en prière, accompagnés des accessoires religieux stéréotypés (Bible, chapelet) dans Prison Break (1.13)

Les prières constituent un autre moyen de consolation et de soutien, et leur occurrence n'est pas rare dans l'échantillon. Ces prières peuvent être tirées de la liturgie chrétienne, la plus fréquente étant: "Le Seigneur est mon berger ${ }^{18}$." Elle exprime la foi dans le salut, la justice, et la protection apportée par Dieu face au mal ${ }^{19}$. On l'entend dans $O Z$ 6.8, Walker, Texas Ranger 2.9 (CBS, 1993-2001), et Prison Break. Le Psaume n'est prononcé que pour l'exécution d'innocents. Promettre un au-delà meilleur pour les malheureux ici-bas peut être interprété comme des paroles de consolation, et/ou de dénonciation de l'injustice. Sont également convoquées des prières libres, improvisées, qui expriment la foi du condamné en un Dieu bienveillant, et en la possibilité de trouver la paix et le pardon, par

${ }^{18}$ Dans la bouche des divers personnages : « The Lord is my shepherd ». Il s'agit du Psaume 23, tiré du Livre des Psaumes.

19 «Le Seigneur est mon berger [...] il me ramène sur le chemin de la justice. [...] Sa houlette est signe de libération et de salut. [...] Il est parole de vie éternelle. » 
exemple dans Cold Case : affaires classées 3.20 (Cold Case, CBS, 2003-2010 ${ }^{20}$ ). On trouve enfin des prières silencieuses, rendues visibles par l'accessoire du chapelet dans À la Maison Blanche 1.14 (The West Wing, NBC, 1999-2006), ou OZ. Elles sont souvent mises en scène au moment même de l'exécution. Ces prières libres et improvisées font écho à la religiosité souvent décrite dans la réalité aux États-Unis, car elles sont non dénominationnelles ${ }^{21}$. La foi exprimée est réduite à l'essentiel, ce qui la rend acceptable par tous les croyants, quelle que soit leur religion. Cette forme basique se retrouve lors des prières publiques, liées à la «religion civile $^{22} »$. Elles témoignent également d'une conception de la religion sans intermédiaire.

Par ailleurs, la peur de l'enfer, évoquée dans de nombreuses intrigues, est dénoncée par l'écrasante majorité d'entre elles. Ainsi, Urgences 14.13 (ER, NBC, 1994-2009), présente le personnage de Robert (Jonathan Banks), un médecin et ancien " bourreau ", ayant pratiqué 17 injections létales. Il est atteint d'un cancer en phase terminale, qu'il interprète comme le signe de sa damnation. Il consacre ses dernières forces à venir en aide aux familles des hommes qu'il a exécutés, comme il le peut (il a sauté dans un lac gelé, pour sauver de la noyade le fils de l'un d'entre eux, raison de sa présence aux urgences). Désespéré et perclus de remords, Robert tente de se suicider, en reproduisant une injection létale dans une salle de soin. Il est interrompu par un médecin, qui tente de le consoler. Robert exige de comprendre le «plan divin » guidant sa destinée, le Dr. Pratt (Mekhi Phifer) lui rappelle alors un des fondements de la foi :

DR. PRATT. Ca [votre vie] n'a pas besoin d'avoir de sens, c'est pour cela que ça s'appelle la foi ${ }^{23}$.

\footnotetext{
${ }^{20}$ Andre Tibbs (Michael Jace), lors de son exécution, fait référence aux derniers mots de la jeune fille pour le meurtre de laquelle il a été condamné, («foi »/«faith ») et dont il est innocent: "Il y a quelque chose d'autre, de plus grand, de meilleur, que ce qui me consume. Et si cette petite fille l'a trouvé, je le trouverai aussi. Il faut que j’y croie, et je trouverai un peu de paix. » (« There's something out there bigger than what's eating me alive, something better. And if that little girl found it, I'll find it too. I've got to believe that, and I'll find some peace. »). Autre exemple dans Prison Break 1. 14: juste avant d'entrer dans la chambre d'exécution, le condamné trouve le courage de rester digne en se répétant comme une litanie qu'il doit avoir la foi ; des flash-back lui remémorent également cette obligation en présentant tous ses proches l'encourageant (« have a little faith » et « take a leap of faith »).

21 L'expression "dénomination religieuse » est une traduction de l'expression anglaise "religious denomination" désignant les confessions, et les différentes branches des Églises. Elles sont particulièrement nombreuses aux États-Unis, comme le montre l'enquête du Pew Research Center citée en introduction. Ce qui est non dénominationnel ne peut donc se rattacher à aucune Église ou aucune croyance en particulier.

22 La "religion civile » est un ensemble de cérémonies et de symboles qui doit unir les Américains autour de l'idée d'une nation inspirée et guidée par Dieu, sans faire référence à une religion ou une dénomination particulière. Elle permet d'associer patriotisme, religion et séparation des Églises et de l'État, voir Froidevaux-Metterie, p. 106 et suivantes : « La religion civile ou la résolution de l'énigme théocratico-laïque »
}

23 «It doesn't have to make sense, that's why it's called faith. » 
On retrouve ici une formule, attribuée à Tertullien : «credo quia absurdum », je crois parce que c'est absurde. Ce rappel semble efficace, puisque Robert renonce à se suicider.

Refus de l'enfer et foi dans le salut soutiennent condamnés, héros et spectateurs : Dieu répare la violence et les injustices en train de se commettre. Une dernière forme de consolation s'exprime par la musique. Trois occurrences sont remarquables : la chanson Hallelujah de Léonard Cohen, dans Cold Case: affaires classées; un chant hébreu dans À la Maison Blanche ; et pour finir, dans Dernier recours, le célèbre hymne : Amazing Grace ${ }^{24}$.

Celles-ci ont plusieurs points communs. Les scènes, baignées de musique extra-diégétique, se trouvent toutes en fin d'épisode. De plus, les scenarii des trois épisodes mettent les personnages principaux en échec (exécution d'un innocent ou probablement tel dans deux cas, échec du président démocrate à faire avancer son pays vers l'abolitionnisme, dans le cas de la série politique). Tout cela donne à ces épisodes une forte charge émotionnelle. La musique exprime la douleur, la tristesse (dans les trois cas, elle évoque des funérailles ${ }^{25}$ ), mais elle apporte aussi un certain apaisement. Pour le spectateur pratiquant, l'utilisation d'une musique à forte tonalité religieuse peut aussi être un rappel du culte ordinaire, souvent accompagné de musique.

Ces fonctions de la musique apparaissent très nettement dans Dernier recours. La fin de l'épisode est composée de deux scènes. La première est la scène d'exécution par injection létale du condamné à mort, Frank Benner (T.K. Carter). Retardé mental, il est probablement innocent. Il a été accompagné pendant tout l'épisode par Sonya Quintano (Marisol Nichols), un des personnages principaux de la série. Celle-ci a assisté à l'ensemble de la préparation pour la mise à mort (du choix des témoins au choix de la méthode d'exécution). Tous les recours ayant échoué, la voilà parmi les témoins, présente pour soutenir Frank. À ses côtés, se trouve la Sœur Quinn, l'accompagnant. Un lent crescendo a cappella débute lors de l'arrivée de Frank dans la chambre d'exécution. La musique devient plus audible à mesure que la mort approche: tous les personnages sont en pleurs, et même les gardiens de prison semblent émus. L'hymne permet de consoler, ses paroles exprimant le bonheur et la délivrance apportés par la foi. Cette signification universelle peut cependant aller de pair avec une interprétation plus politique. En effet, Frank est afro-américain, or cet

${ }^{24}$ Hymne chrétien, paroles de John Newton, ministre anglican, publiées pour la première fois en 1779, sur une musique de William Walker composée en 1835 (accompagnant originellement un chant nommé New Britain)

${ }^{25}$ La chanson de Léonard Cohen (publiée en 1984) est diffusée pendant les funérailles de l'exécuté, le chant hébreu est un chant de funérailles et Amazing Grace est souvent utilisé lors des funérailles. 
hymne est également associé aux Églises noires. Le racisme dans la justice et la pratique de la peine capitale sont en effet un sujet de débat dans la société ${ }^{26}$. À l'issue de cette scène, où la musique a joué un rôle de soutien, un moment de silence est laissé en suspens, pour contempler la mort donnée.

Débute ensuite la deuxième scène, qui sert d'épilogue. En effet, comment continuer après avoir assisté à un tel drame? À une telle injustice? Cette série, diffusée sur $\mathrm{ABC}$, chaîne familiale, ne peut se clore sur un échec aussi douloureux. La scène suivante tente d'atténuer la peine et la tristesse de l'entourage du condamné, ici limité à Sonya, qui sert de relais au spectateur. Un gardien lui donne le carnet à dessin de Frank, et la mélodie de l'hymne reprend en toile de fond. Ce carnet rappelle le caractère enfantin du condamné, et donc sa possible innocence. Le dessin représente un avion volant dans un ciel radieux : la musique et les images convergent pour suggérer au spectateur que le condamné est parti pour un monde meilleur, il est libre et ne souffre plus. Sonya esquisse un petit sourire mouillé de larmes : c'est le début de la consolation et du deuil. Sonya, et le spectateur, peuvent reprendre leur chemin.

Les «traces religieuses» sont ici instrumentalisées, pour représenter la peine de mort comme une épreuve, et pour montrer comment y faire face et s'en remettre. Cet appel à la religion entre aussi dans le cadre de représentations plus communes pour marquer les grands événements de la vie : baptême, mariage, et passage de vie à trépas - dernière expérience qui est bien celle des condamnés à mort et de leurs proches.

\section{Les « traces religieuses » comme moyen de ritualisation et de sacralisation?}

La présence de «traces religieuses » peut conforter/renforcer le protocole de l'exécution, et parfois même le sacraliser par des actes fortement ritualisés.

En effet, du personnel religieux est présent jusque dans la chambre d'exécution (Walker, Texas Ranger, OZ). Ce personnel y accomplit des actes religieux comme la bénédiction du condamné, ou la déclamation de prières. Ainsi, dans $X$-Files : Aux frontières du réel 1.13 (The $X$ Files, FOX, 1993-2002), la scène d'exécution dans la chambre à gaz a lieu en présence d'un prêtre, qui se trouve dans la salle des témoins avec le directeur de la prison. Alors que le gaz se répand dans la chambre d'exécution, le prêtre récite le « Notre Père ». Il est difficile

\footnotetext{
${ }^{26}$ Un Rapport du DPIC (Death Penalty Information Center) en témoigne: Richard C. Dieter, The Death Penalty in Black and White: Who Lives, Who Dies, Who Decides, DPIC, juin 1998
} 
de ne pas identifier la mort de l'exécuté avec les paroles de cette prière ( «Que Ta Volonté soit faite ${ }^{27} »$ ).

Ceci explique pourquoi cette participation à la procédure est critiquée dans certaines intrigues. Par exemple, l'épisode 13.21 de la série animée Les Simpsons (The Simpsons, FOX, 1989-) dépeint un clergé indigne de ses fonctions, qu'il soit catholique ou protestant: alors que le prêtre refuse de soutenir, lors de leur attente dans les couloirs de la mort, Marge et Homer, parce qu'ils ne sont pas catholiques, le révérend se bat à coups de Bible dans la prison avec ledit prêtre. Boston Justice 1.17 (Boston Legal, ABC, 2004-2008), présente un aumônier de prison, qui déclare cyniquement à l'avocat du condamné, lui demandant son aide lors du dernier recours : « Le clergé est présent uniquement pour faire en sorte que le condamné soit prêt et aille volontairement à la mort ${ }^{28}$. »

Les fictions de David E. Kelley, abolitionniste bien connu, utilisent le thème récurrent de la religion comme «opium du condamné », instrumentalisée pour manipuler les consciences. Dans le cas de cet épisode, le condamné est faible d'esprit. Il est passé aux aveux alors même s'il ne se souvient pas avoir commis le crime dont il est accusé. Il a été persuadé par l'aumônier qu'il ira en enfer s'il rétracte ses aveux, et qu'il doit être stoïque face à la mort.

La ritualisation et la sacralisation de l'exécution semblent culminer avec la reproduction de la crucifixion. En effet, des images troublantes sont mises en scène dans le cas d'exécutions par injection létale. Des exécutés sont montrés " mis en croix 》 et comme crucifiés dans six épisodes : Les Experts 3.6 (CSI, CBS, 2000-), Urgences, Six Feet Under 3.12 (HBO, 2001-2005), Cold Case : affaires classées, À la Maison Blanche, The Good Wife 5.1. La position est proche, bien que moins mise en valeur, dans trois autres : Lie to Me 2.19 (FOX, 20092011), Boston Justice, Walker, Texas Ranger. En tout, sur les 12 exécutions par injection létale montrées à l'écran, neuf utilisent l'image de la crucifixion. Il s'agit donc bien d'un topos des représentations de l'injection létale. Comment l'interpréter?

On peut, tout d'abord, émettre l'hypothèse d'un simple rapport avec la réalité. Les photographies de tables d'exécutions réelles nous suggèrent que cela n'est pas invraisemblable (figure 3). Cependant, toute prise de vue d'une exécution étant formellement interdite, il nous est impossible d'affirmer que, dans la réalité, les exécutés sont effectivement et systématiquement «mis en croix». Cette position n'est par ailleurs pas rare lors de certaines opérations chirurgicales, or l'injection létale mime le protocole médical.

\footnotetext{
27 « Thy will shalt be done », il s'agit d'une prière enseignée par le Christ lui-même à ses apôtres selon les Évangiles : Matthieu, $6: 9$-13 et Luc, $11: 2-4$

28 « Role of clergy is only to get the inmate ready and willing to die. »
} 


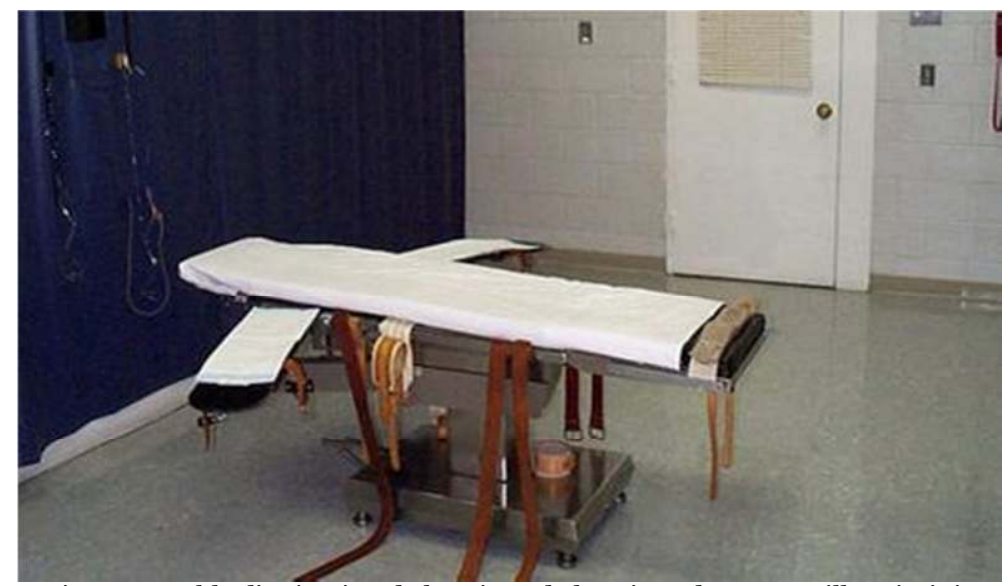

Fig. 3 : La table d'exécution de la prison de la prison de Greensville, Virginie (photographie New York Times)

Que ces crucifixions soient conformes à la réalité ou non, leur mise en scène instrumentalise de fait un repère religieux et culturel fondamental. Mettre le condamné dans la position du Christ prête à l'exécution une dimension symbolique qui interpelle. En effet, cette image peut être comprise comme une sacralisation de l'exécution, ou au contraire, comme une critique, en lui donnant un caractère sacrilège.

Certains éléments récurrents semblent suggérer une valeur critique. En effet, cinq de ces exécutions avec mise en croix se déroulent dans des épisodes critiques vis-à-vis de la peine de mort, voire abolitionnistes ${ }^{29}$. Les quatre autres épisodes ont pour point commun de présenter des exécutions de condamnés dont la culpabilité n'est pas établie aux yeux du spectateur, le plus souvent parce que celles-ci ont lieu en ouverture d'épisode, alors que le spectateur ignore tout de l'affaire ${ }^{30}$.

L'impact de ces exécutions illégitimes, voire injustes, aux yeux du spectateur ${ }^{31}$, est donc renforcé par la mise en croix. Cette corrélation semble confirmée par la comparaison des deux exécutions ayant lieu dans l'épisode des Experts de l'échantillon. La première est la scène

29 Il s'agit des épisodes suivants : Urgences, Cold Case : affaires classées, À la Maison Blanche, The Good Wife 5.1, Boston Justice. Par ailleurs, dans trois d'entre eux, les condamnés sont certainement ou probablement innocents.

${ }^{30}$ Il s'agit des épisodes suivants : Les Experts, Six Feet Under, et Lie to Me. Walker, Texas Ranger constitue un cas un peu à part : la culpabilité du condamné apparait douteuse tôt dans l'épisode. Son innocence est définitivement établie alors que les produits mortels sont sur le point d'être injectés dans son bras.

${ }^{31}$ Sur les neuf personnages crucifiés, un seul est coupable de manière certaine au moment de sa crucifixion (celui de la série À la Maison Blanche). 
d'ouverture : l'affaire est inconnue du spectateur, le condamné est ramené à la vie après l'injection (donc ressuscité), car il y a un doute sur sa culpabilité. La mise en scène du condamné, posé en croix sur la table d'exécution, est valorisée par un plan en plongée (figure 4).

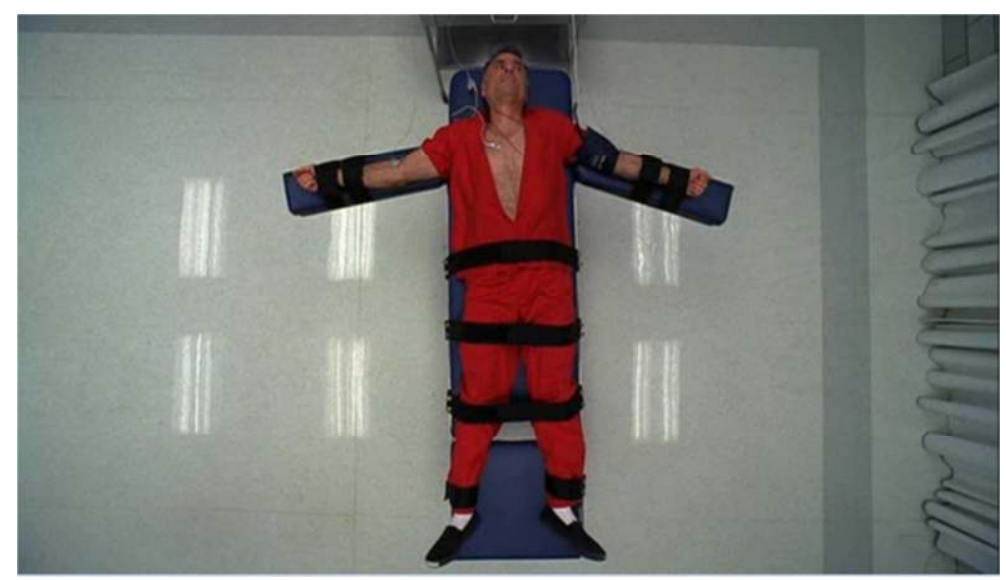

Fig. 4 : Vue en plongée du condamné lors de la première exécution, une mise en croix mise en valeur par la mise en scène (Les Experts, 3.6)

La seconde exécution, à la fin de l'épisode, quant à elle est conforme aux stéréotypes : la culpabilité ne fait aucun doute, l'exécuté est un criminel monstrueux qui a tué une jeune fille innocente. Le rideau s'ouvre, révélant la chambre d'exécution aux témoins, connus du spectateur. Cette fois-ci, le choix de mise en scène ne met pas en valeur la position du corps du condamné ${ }^{2}$. Les plans montrent un homme allongé et attaché (figure 5).

${ }^{32}$ Les trois seules exécutions qui ne donnent pas lieu à une mise en croix ont pour point commun de se dérouler en fin d'épisode. 


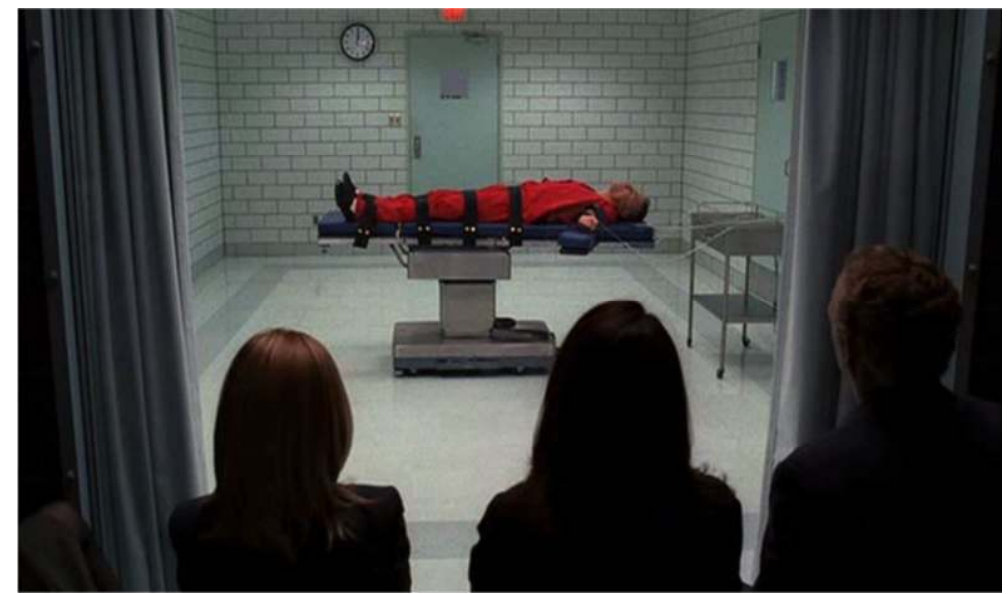

Fig. 5 : L'exécution de fin d'épisode, un choix de mise en scène sans dimension symbolique religieuse (Les Experts, 3.6)

\section{Les « traces religieuses » comme arguments d'autorité dans le débat sur la peine de mort ?}

Les «traces religieuses » peuvent aussi être utilisées dans le débat sur la peine de mort pour valider une opinion, son origine sacrée la rendant indiscutable.

Un lieu commun de la représentation de la peine de mort est la manifestation devant les portes de la prison, peu avant une exécution. Les arguments sur les panneaux des manifestants sont ainsi formulés en termes religieux, érigés en autant de prescriptions : " Tu ne tueras point » (Prison Break), " Dieu doit être le seul juge » (Walker, Texas Ranger), ou « L'enfer pour les assassins 33 » (Criminal Minds 1.14, CBS, 2005-).

Les Écritures sont également utilisées de manière régulière par le biais de citations. L'épisode de Criminal Minds s'ouvre avec un extrait de la Genèse, énoncé en voix-off : "Qui verse le sang de l'homme, par l'homme aura son sang versé34. " Autre exemple dans The Practice 2.10 (ABC, 1997-2004) : des passants sont interrogés par un journaliste sur leur opinion concernant la peine de mort. L'un d'entre eux rappelle la loi du talion (« œil pour œil ») comme s'il s'agissait d'une évidence mettant fin à toute discussion.

La validation d'une opinion par l'utilisation de références religieuses semble un topos des représentations, mais la critique de ce

33 Respectivement : « Thou shalt not kill », « Let God be the judge », et « Hell to killers ».

34 « Who sheddeth the man's blood by man shall his blood be shed. », Genèse, 9-6 
phénomène est menée dans quelques épisodes. Par exemple, dans The Good Wife (3.8), un prêtre plaide la cause d'un homme qui a enlevé, tué et violé deux jeunes adolescentes. Le condamné est tout à fait antipathique, et refuse de se repentir. Le prêtre, interrogé par un des avocats, formule la règle absolue : il ne doit pas être tué. Il n'apporte aucun complément à cet argument d'autorité. En retour, l'héroïne, qui identifie les victimes à sa propre fille, exprime un doute sur le bienfondé de cet absolu.

La critique de l'instrumentalisation des textes sacrés dans le débat sur la peine de mort est la plus aboutie dans À la Maison Blanche, par le caractère savant des arguments échangés. Le président, démocrate, doit choisir d'utiliser ou non son droit de grâce, un condamné à mort fédéral étant sur le point d'être exécuté. Dans cet épisode, il ne s'agit pas de critiquer une règle absolue mais, plus fondamentalement, de nier la possibilité même de tirer des Écritures une assurance définitive quant à la légitimité de la pratique du châtiment suprême. En effet, chacun peut y voir ce qu'il veut, et sélectionner les passages qui justifient son point de vue. Cette démonstration est menée par plusieurs personnages, et notamment par un rabbin, que le directeur de la communication de la Maison Blanche, Toby Ziegler (Richard Schiff), vient consulter.

LE RABBIN. [La Torah] dit qu'un enfant rebelle peut être amené devant les portes de la ville pour y être lapidé. Elle dit que l'homosexualité est une abomination qui doit être punie de mort. Elle dit que les hommes peuvent être polygames et que l'esclavage est acceptable. D'après moi, ce texte reflète la sagesse de son temps. Mais ces principes sont tout simplement indéfendables au regard des valeurs d'aujourd'hui 35 .

Les «valeurs d'aujourd'hui » se sont donc sécularisées selon le rabbin, au point de rendre loi du talion tout aussi obsolète que celle qui autorise l'esclavage. Les partisans de la peine de mort qui s'appuient sur la Bible choisissent d'ignorer un certain nombre de prescriptions religieuses, ils opèrent donc un tri.

Autre exemple, le cinquième commandement, dont la traduction est discutée par le président Bartlett (Martin Sheen) lui-même : « Le commandement ne dit pas : «tu ne tueras point», il dit: «tu ne commettras pas de meurtre ${ }^{36}$. » Selon la traduction adoptée, le texte peut parfaitement servir aux deux camps : l'utilisation du verbe tuer («to kill») interdit la peine capitale, le verbe assassiner («to murder ») n'interdit que le meurtre, ce qui autorise la peine de mort.

35 « [The Torah] says a rebellious child can be brought to the city gate and stoned to death. It says homosexuality is an abomination and punishable by death. It says men can be polygamous and slavery is acceptable. For all I know, that thinking reflected the best wisdom of its time. But it's just plain wrong by any modern standard. »

${ }^{36}$ " The commandment doesn't say : 'thou shalt not kill', it says : 'thou shalt not murder'. » 
Cet épisode est également original car construit sur un « fil rouge religieux », unique dans l'échantillon. En effet, les scénaristes multiplient la confrontation de personnages avec des représentants de plusieurs religions identifiées (le spectateur entend ainsi le point de vue sur la peine capitale d'un rabbin, d'une quaker et d'un prêtre). Des arguments politico-religieux sont avancés. Le président, qui décide finalement de ne pas accorder la grâce, le fait pour des raisons politiques et religieuses : catholique, il ne doit pas être soupçonné d'être influencé par sa religion. Il prendrait le risque d'être accusé par ses opposants d'être soumis à la papauté. En effet, un président américain ne peut obéir qu'à la constitution ${ }^{37}$.

Le choix de ce fil conducteur est fait dans un contexte historique particulier. Cet épisode est diffusé pour la première fois en septembre 2000, au moment de la campagne présidentielle de G.W. Bush, sur laquelle pèse l'influence de la droite chrétienne, évangélique et fondamentaliste. En démontrant que les Écritures sont instrumentalisées, l'épisode critique l'arsenal politico-religieux déployé depuis les années Reagan par le courant évangélique ${ }^{38}$. Les arguments de la droite chrétienne sont retournés contre leurs créateurs, ce qui donne à cet épisode un caractère militant, pro-démocrate (conforme aux opinions bien connues du créateur de la série, Aaron Sorkin).

\section{Les « traces religieuses » comme procédé visuel et rhétorique}

Les «traces religieuses » sont aussi présentes grâce à la mise en scène, qui peut infléchir le sens de la fiction. Le premier procédé rhétorique, souligné par des effets visuels, réside dans la déviation finale du sens. Deux épisodes de séries à « dispositif divin 39 » entrent dans ce schéma narratif : Code Quantum 3.19 (Quantum Leap, NBC, 1989-1993) et Ghost Whisperer 1.14 (CBS, 2005-2010). Ces deux épisodes sont plutôt critiques vis-à-vis de la peine de mort, jusqu'à l'épilogue. En effet, dans les deux cas, la culpabilité des condamnés est douteuse. De plus, ces deux séries fantastiques mettent en scène l'exécution des héros eux-mêmes, de façon particulièrement terrifiante

\footnotetext{
${ }^{37}$ La crainte d'un président dominé par une influence extérieure a notamment refait surface lors de la campagne de John Fitzgerald Kennedy. Son discours devant l'Association des pasteurs de Houston (Texas), du 12 septembre 1960, vise à rassurer une partie de l'opinion " antipapiste »; il déclare par exemple : « Je crois dans une Amérique où la séparation de l'Église et de l'État est absolue, où aucun prélat catholique n'ordonnerait à un président (fût-il catholique) comment il doit agir [...]. " ( I I believe in an America where the separation of church and state is absolute, where no Catholic prelate would tell the president (should he be Catholic) how to act [...]. »)

${ }^{8} 8$ Voir Froidevaux-Metterie, p. 84 et suivantes : « L’influence de la droite chrétienne sur la politique américaine »

39 Nous désignons ainsi des séries fantastiques dans lesquelles les héros sont envoyés par une « force bienveillante » ou disposent d'un don de même origine (visions, communication avec les morts, ...), pour faire le bien.
} 
(une exécution sur la chaise électrique, en ouverture d'épisode, pour le voyageur dans le temps, et une pendaison pour la médium). La critique de la peine de mort est plus marquée dans Code Quantum où le procureur instrumentalise les exécutions, de façon très cynique, pour faire avancer sa carrière - quitte à manipuler les preuves et intimider les témoins gênants. Dans les deux intrigues, au moment de l'épilogue, temps de la morale, un retournement de situation est opéré : le condamné est coupable. La morale est sauve, aucun innocent n'est exécuté.

Les «traces religieuses» entrent ici en jeu, en participant au happy end final. Dans Ghost Whisperer, le pendu " entre dans la lumière », c'est-à-dire au paradis (au prix d'une certaine incohérence, puisqu'il s'agit d'un meurtrier). Dans Code Quantum, le "méchant » est puni et le héros réalise son saut habituel dans le temps : la mission, confiée par la divinité, a été accomplie. Ici, le happy end a lieu pendant l'exécution même. Le héros fait toutes les révélations nécessaires alors qu'il est sanglé sur la chaise électrique. Ses dernières paroles sauvent donc les personnages méritants (son coaccusé, innocent, et l'assistante du procureur qui l'a soutenu). Il y a un détournement du sens, mais également du regard, au sens propre du terme. La mise en scène suit le héros qui fait son saut dans le temps, il passe d'un corps à l'autre et quitte la chaise électrique pour un ring de boxe. Par conséquent, le spectateur n'assiste pas à la mort du condamné, qui est escamotée (figures 6, 7, et 8). Exécution et happy end vont de pair, suggérant que la divinité ne s'oppose pas à l'exécution des coupables.

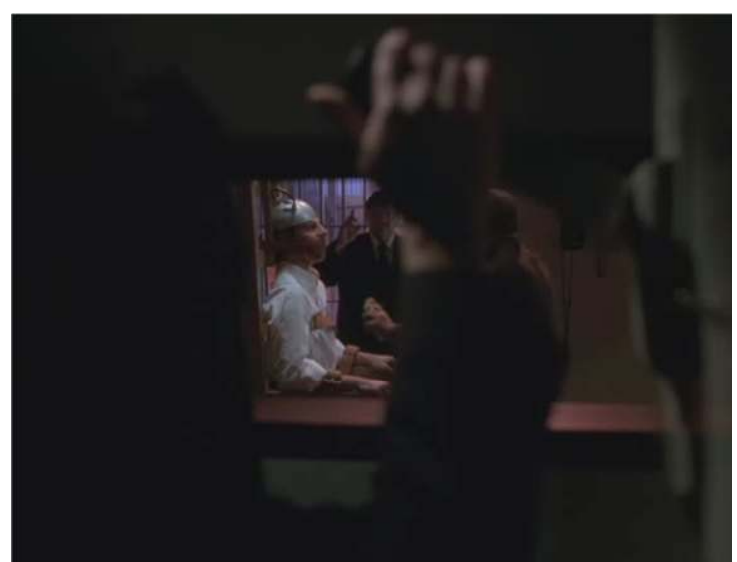

Fig. 6 : Sam Beckett (Scott Bakula) sur la chaise électrique (Code Quantum, 3. 19) 


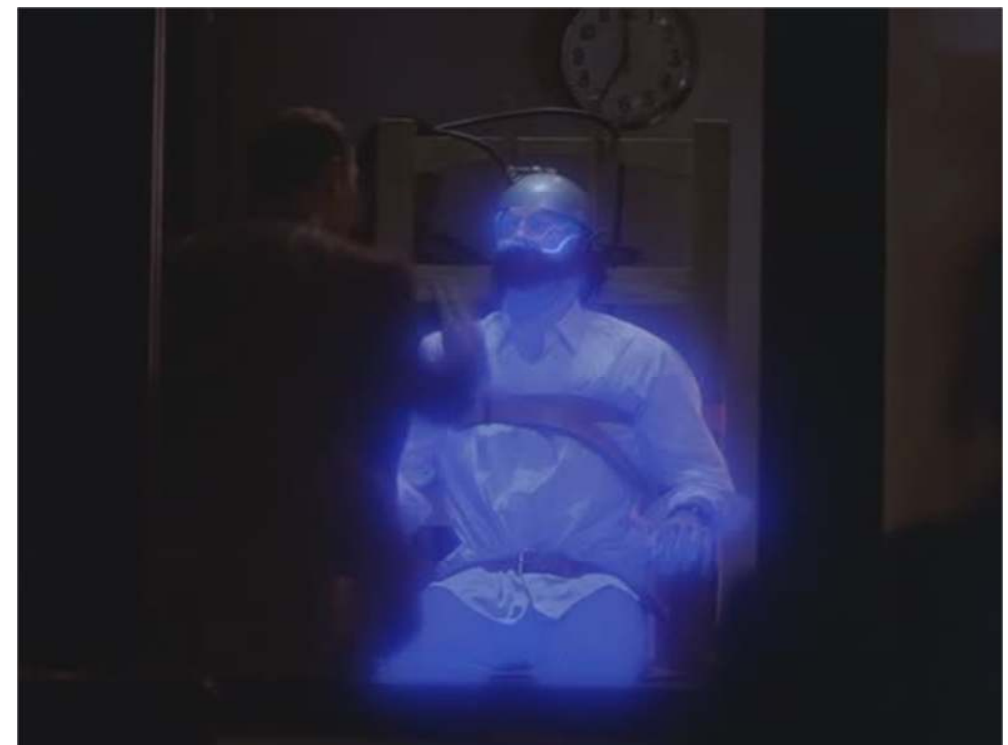

Fig. 7 : Alors que l'électrocution commence, le saut dans le temps est toujours en cours (Code Quantum, 3. 19)

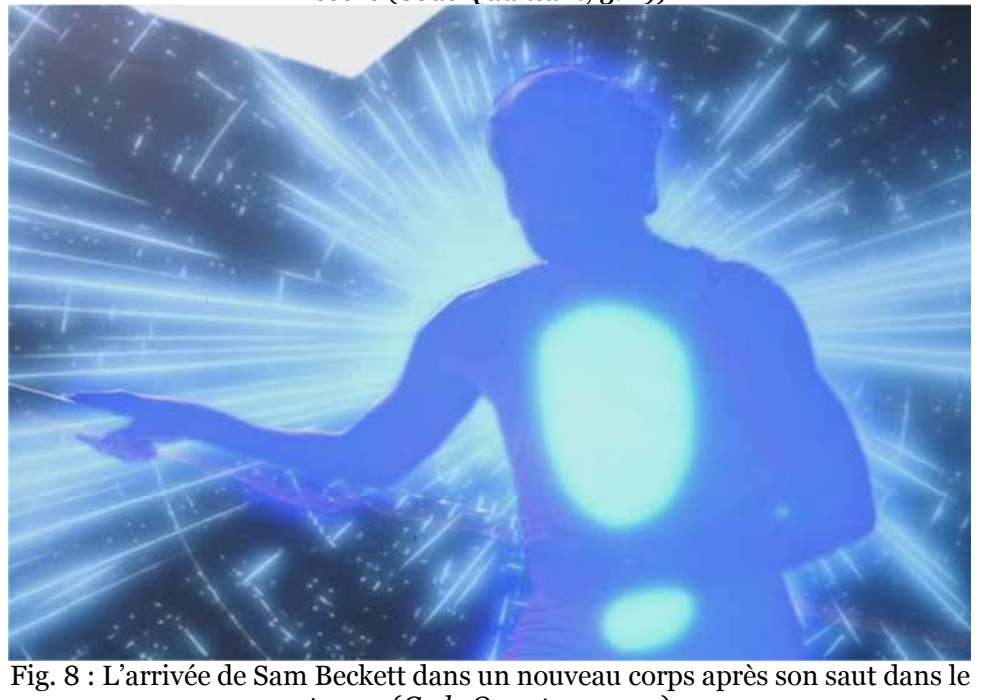
temps (Code Quantum, 3. 19)

Un dernier procédé rhétorique et visuel apparaît dans À la Maison Blanche, par accumulation des images et des symboles. Le spectateur 
assiste à l'exécution en quelques plans très brefs, où les images se superposent en fondus enchaînés : le condamné en position de crucifié, la mère du condamné et le président tous deux en prière, chapelet à la main (figures 9 et 10). Le sacrifice reproduit ne semble entraîner que de la douleur, point commun des trois personnages entremêlés à l'écran. De plus, le chef d'État, du fait de la superposition des images, semble présent dans les deux lieux en même temps (la Maison Blanche, et la chambre d'exécution).

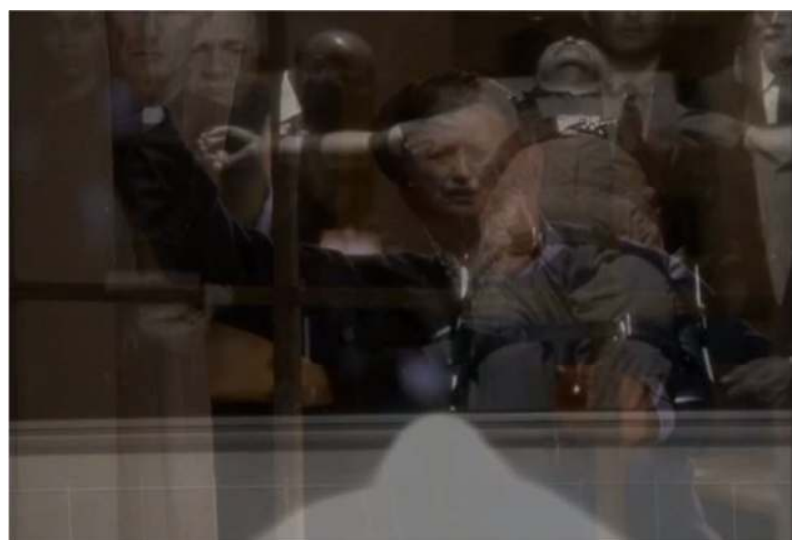

Fig. 9 : Le condamné, sa mère et le président entremêlés lors de l'exécution (À la

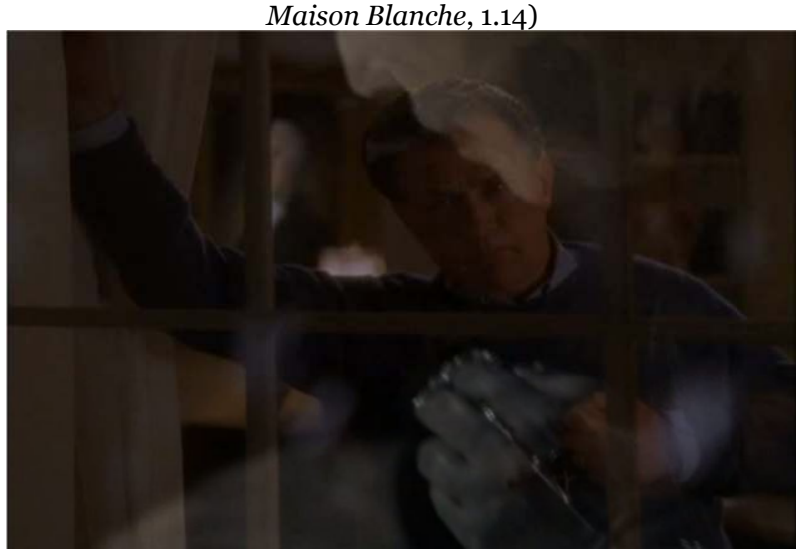

Fig. 10 : Fin du plan débuté par la figure 9, la mère du condamné en noir et blanc et le président (Á la Maison Blanche, 1.14)

Ce dédoublement se poursuit également dans la scène suivante, qui est aussi l'épilogue. Durant tout l'épisode, le président Bartlett est mis en scène « en majesté », en tant que figure politique. Confronté à 
un choix difficile, il se renseigne sur le condamné, consulte son entourage et ses conseillers, pour parvenir à une décision. Dans l'épilogue, il se dédouble : il est à la fois l'institution («the office ») et l'individu (Jed Bartlett, catholique sincère). Cette scène se déroule dans le bureau ovale, où le président reçoit son confesseur. L'attachée de presse de la Maison Blanche entre et donne au chef d'État, sans dire un mot, une note annonçant la mort du condamné. Le prêtre rompt le silence en demandant au président s'il veut être entendu en confession. La réponse, positive, se fait un peu attendre. Bartlett, chapelet à la main, se met à genoux sur le blason des États-Unis (figure 11). Il reconnaît sa culpabilité dans la mort d'un homme. Mais, en tant que représentant élu du peuple, cette confession est aussi prononcée en son nom. La responsabilité est double : celle de l'homme et celle de l'État.

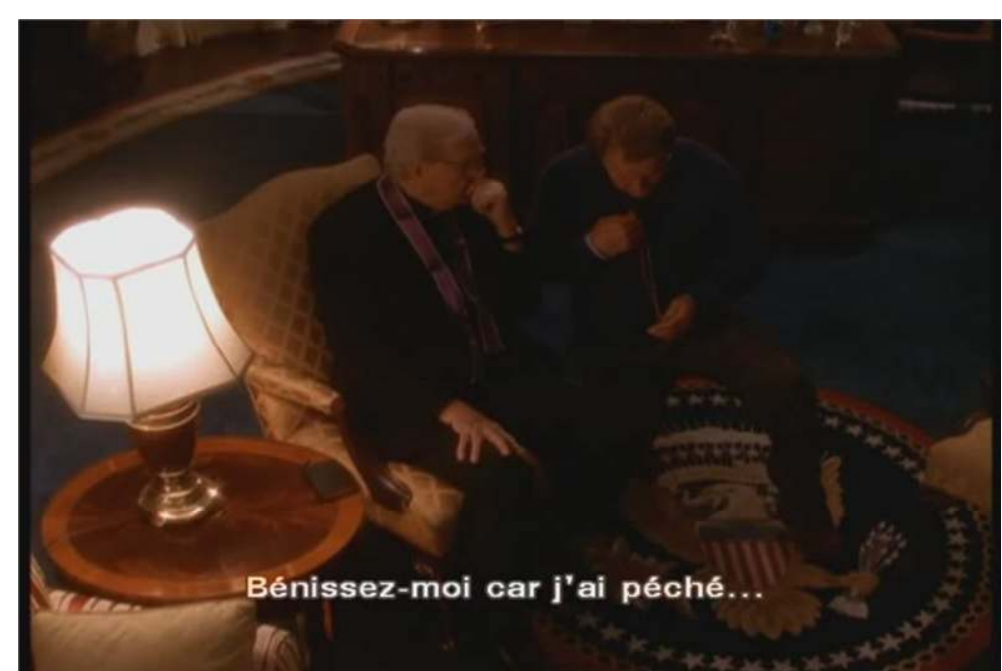

Fig. 11 : La confession du président Bartlett, un acte personnel et politique par le choix du décor et de la mise en scène

(À la Maison Blanche, 1.14)

En conclusion, les rapports entre la religion et la peine de mort sont multiples et complexes. Les « traces religieuses » sont présentes lors du processus concret de la mise à mort par le biais de personnages (prêtres, ministres), d'actes sacrés (bénédictions, prières), de dialogues (dernières paroles des condamnés), d'accessoires (chapelet) et de choix de mise en scène (crucifixion). Il s'agit d'un topos de la représentation du protocole de l'exécution. Ainsi, dans l'ensemble du corpus, parmi les 35 exécutions menées à leur terme à l'écran, 22 sont accompagnées d'éléments religieux dans la phase de préparation et/ou dans la phase finale. Une partie de ces éléments semblent instrumentalisés pour 
valider la pratique de la peine de mort, en prêtant une dimension sacrée au rituel. Cependant, même dans le cas des épisodes les moins critiques, la religion est une instance de légitimation qui coexiste avec d'autres arguments, souvent plus explicites : la pratique de la peine de mort est légale, c'est un châtiment approprié pour un criminel monstrueux, le système judiciaire est efficace et punit les coupables, ... De même, les rites religieux, mis en scène lors du protocole de l'exécution, en accompagnent d'autres, séculiers (choix des témoins, dernière visite médicale, dernier repas, ...).

Ces éléments nous paraissent donc comme autant d'indices d'une sécularisation de la représentation de la peine de mort, la présence de la religion lors des exécutions étant courante, mais pas prépondérante. De plus, la question de la foi est discutée explicitement dans un nombre très réduit d'épisodes (celui de la série Urgences se détache par son ton inquiet et douloureux). La religion le plus souvent représentée nous semble donc relever surtout d'une forme de pratique sociale.

Enfin, les «traces religieuses » sont présentes lors des débats sur la peine capitale représentés à l'écran. Évocation rapide de la loi du talion, ou du respect sacré de la vie, emploi de slogans se référant à Dieu, les arguments religieux sont utilisés par les deux parties. Toutefois, dans tout le corpus, un seul épisode met en scène un débat approfondi sur la peine de mort en termes religieux (À la Maison Blanche). D'ailleurs, même au sein de celui-ci, le fil rouge religieux est complété par des questions séculières comme celle de la vengeance, du respect de la procédure judiciaire, ou de l'exception américaine parmi les grandes démocraties concernant la pratique de la peine capitale. Ceci nous paraît constituer un autre signe de sécularisation, touchant cette fois-ci le débat. Les questions religieuses ne sont pas en tête des priorités, dans la fiction comme dans la réalité. En effet, ce sont les problèmes pratiques de la mise à mort qui sont les plus discutés aujourd'hui (en particulier le protocole de l'injection létale ${ }^{40}$ ). Ceci se traduit dans la fiction télévisée par des intrigues évoquant les tests ADN (Boston Justice, The Practice 4.20, mais aussi Les Experts par exemple). Quelques-unes mettent en scène des injections létales qui se déroulent mal, que le mauvais état veineux du condamné soit en cause (The Good Wife 5.1) ou qu'il s'agisse d'une perfusion mal posée (Urgences, New York, Police Judiciaire 18.941 (Law \& Order, NBC, 1990-2010)). En écho à l'actualité, certaines fictions présentent des exécutions retardées, voire annulées, faute de produits pour l'injection (The Good Wife 2.9 et 5.1).

$4^{40}$ En septembre 2007, la Cour suprême des États-Unis suspend toutes les exécutions par injection létale, le temps d'examiner la constitutionnalité du protocole utilisé ; en avril 2008, l'arrêt Baze v. Rees le valide.

${ }^{41}$ Ces deux derniers épisodes sont d'ailleurs diffusés pour la première fois pendant le moratoire de la Cour suprême, respectivement en janvier et février 2008. 
Instrumentalisée pour valider et/ou critiquer la peine de mort, la religion constitue un des topoï attachés à la représentation de ce thème. On peut donc avancer l'hypothèse de sa relative et partielle sécularisation. L'étude du corpus montre qu'un des premiers épisodes présentant le débat sous sa forme classique (opposant contre partisan) est diffusé pour la première fois en 1992. Il s'agit de Vengeance (New York, Police Judiciaire 2.16). Le procureur y avance déjà des arguments pragmatiques (comme le coût de la procédure) face à son assistant, qui évoque la loi du talion. La sécularisation du débat nous semble donc le résultat de l'abandon, par les abolitionnistes fictifs et réels, des grandes questions de principes. Ainsi, lors du débat organisé par le Pew Forum en 2002, évoqué plus haut, le sénateur Paul Simon répond à Justice Scalia (qui s'est appuyé sur la Bible pendant son intervention) en utilisant presque uniquement des arguments techniques et pragmatiques.

Cette hypothèse d'une sécularisation partielle et relative du thème de la peine de mort est confirmée par le discours le plus entendu dans l'échantillon: la peine de mort est le châtiment mérité en cas de meurtre. Cette formulation, bien que dégagée de références religieuses, semble un dérivé sécularisé de la loi du talion (" une vie pour une vie »). Or dans un sondage réalisé en 2011, plus de $50 \%$ des défenseurs de la peine de mort ont recours à cet argument 42 . On peut y voir une référence religieuse implicite, ancrée dans les mentalités, dont il est inutile d'utiliser la forme scripturaire pour la rendre explicite.

\section{Les auteurs}

Julie RICHARD est professeur agrégé d'histoire au Lycée Alfred-Kastler (Cergy-Pontoise, Val d'Oise). Elle a réalisé un mémoire sur La représentation des Anciens Combattants de la Grande Guerre dans les films français de fiction, 1919-1989 (2002). La mise en place d'un atelier pédagogique sur la représentation de la peine de mort dans les séries télévisées américaines, en collaboration avec Georges Caron, les a mené à préparer un ouvrage de synthèse sur ce sujet.

Georges CARON est professeur certifié d'histoire-géographie au Lycée AlfredKastler, et au Lycée de la Nouvelle Chance. Spécialiste de la Sicile médiévale, il prépare, avec Julie Richard, un ouvrage sur la représentation de la peine de mort dans les séries américaines.

42 Étude menée du 9 au 14 novembre 2011 par le Pew Research Center auprès de 1222 personnes favorables à la peine de mort. 